\title{
Editorial zum Themenschwerpunkt Pfannenrevisionen
}

Beim Wechsel von gelockerten Pfannen müssen mehrere Ziele erreicht werden. Das Drehzentrum des Hüftgelenks soll wieder rekonstruiert werden, ebenso die Pfannenkontinuität und -integrität. Das neue Implantat soll eine solide Primärfixation erreichen und keine Migration verursachen. Das Implantat sollte es erlauben, einen bestehenden Knochendefekt möglichst durch Knochentransplantation zu verkleinern, bzw. zumindest vermeiden, dass ein weiterer Knochenverlust entsteht.

Für die Revision von gelockerten Pfannen stehen verschiedene Konzepte bzw. Revisionssysteme zur Verfügung (Tabelle 1).

\section{Tabelle 1}

Verschiedene Konzepte für den Pfannenwechsel.

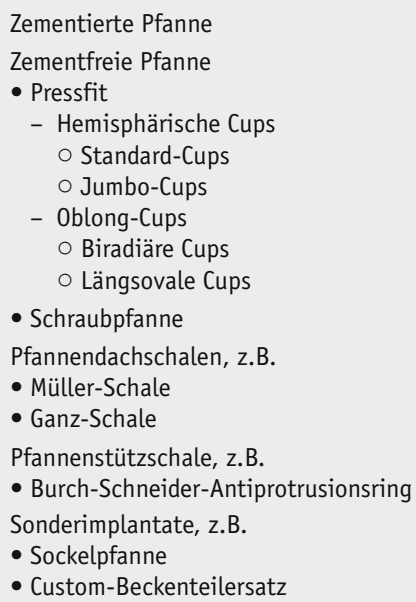

Bei Pfannenrevisionen ist es generell wichtig, mehrere Revisionsimplantate vorrätig zu haben, da es anhand der präoperativen Röntgenaufnahmen nicht immer gelingt, die Defektausdehnung richtig einzuschätzen, und sich der Defekt häufig intraoperativ nach dem Débridement des Lockerungsgewebes größer darstellt als präoperativ vermutet. Die verschiedenen Revisionsimplantate haben ihre defektspezifischen Stärken und Schwächen. Zur Abdeckung der meisten Defektsituationen sind drei Revisionspfannensysteme notwendig. Bei kleineren kavitären und auch segmentalen Defekten (Paprosky I sowie IIa und IIb) reichen in der Regel Primärpfannen bzw. Pfannendachschalen aus, bei mittleren Defekten (Paprosky IIb und IIc) sind zementfreie Revisionsimplantate bzw. Pfannendachschalen und bei größeren Defekten (Paprosky IIIa und IIIb) häufig Pfannenstützschalen bzw. Sonderimplantate (Sonderanfertigungen oder Trabecular-MetalPfannen mit Augments) notwendig. Bei Beckendiskontinuitäten sind häufig zusätzliche Osteosynthesen oder die Verwendung von Sonderanfertigungen indiziert.

In diesem Schwerpunktheft sollen vier Revisionsprinzipien erläutert werden. Jedes hat seine Vor- und Nachteile und seine defektspezifische Indikation, die im Folgenden dargestellt werden. Insgesamt ist diesen Operationen gemeinsam, dass sie einer nicht zu unterschätzenden Erfahrung des Operateurs bedürfen und dieser stets in der Lage sein muss, intraoperativ auftretende Komplikationen zu beherrschen.

Bernd Fink 